PROCEEDINGS OF THE

AMERICAN MATHEMATICAL SOCIETY

Volume 137, Number 7, July 2009, Pages 2217-2224

S 0002-9939(09)09882-7

Article electronically published on March 4, 2009

\title{
BILINEAR SUMS WITH EXPONENTIAL FUNCTIONS
}

\author{
IGOR E. SHPARLINSKI
}

(Communicated by Wen-Ching Winnie Li)

\begin{abstract}
Let $g \neq 0, \pm 1$ be a fixed integer. Given two sequences of complex numbers $\left(\varphi_{m}\right)_{m=1}^{\infty}$ and $\left(\psi_{n}\right)_{n=1}^{\infty}$ and two sufficiently large integers $M$ and $N$, we estimate the exponential sums$$
\sum_{\substack{p \leq M \\ \operatorname{gcd}(a g, p)=1}} \sum_{1 \leq n \leq N} \varphi_{p} \psi_{n} \mathbf{e}_{p}\left(a g^{n}\right), \quad a \in \mathbb{Z},
$$

where the outer summation is taken over all primes $p \leq M$ with $\operatorname{gcd}(a g, p)=1$.
\end{abstract}

\section{INTRODUCTION}

Let us fix an integer $g \neq 0, \pm 1$. Various questions concerning the distribution of residues of the exponential function $g^{x}$ in residue rings when $x$ takes consecutive integer values and also when it runs through some general and special sequences (such as smooth or prime numbers) have always been intensively studied; see 1, 2, 4, 5, 6, 7, 8, 9, 10, 11, 12, 13, 14, 17, and the references therein. For example, for $g=2$ they have a natural interpretation as results about the distribution of Mersenne numbers in residue classes; see 1, 4, 11, 12. They are also related to various questions about the distribution $g$-ary digits of rational fractions; see [13, 14. Furthermore, these results also have various applications to such areas as cryptography and pseudorandom number generators; see [16, 18]. Most of the applications are based on estimates of corresponding exponential sums.

More precisely, for an integer $m \geq 1$ and a complex $z$, we define

$$
\mathbf{e}_{m}(z)=\exp (2 \pi i z / m) \text {. }
$$

Several estimates have recently been obtained for exponential sums

$$
\sum_{1 \leq \ell \leq N} \mathbf{e}_{p}\left(a g^{\ell}\right), \quad a \in \mathbb{Z},
$$

over primes $\ell \leq N$; see [1, 4. Furthermore, in [11, 12] more general sums

$$
\sum_{k=1}^{K} \mathbf{e}_{p}\left(a g^{s_{k}}\right), \quad a \in \mathbb{Z}
$$

Received by the editors September 17, 2008.

2000 Mathematics Subject Classification. Primary 11L07, 11L26.

During the preparation of this paper, the author was supported in part by ARC grant No. DP0556431.

(C)2009 American Mathematical Society Reverts to public domain 28 years from publication 
have been estimated on average over $p \leq M$, for arbitrary sequences of integers $\mathcal{S}=$ $\left(s_{k}\right)_{k=1}^{\infty}$, provided that $\mathcal{S}$ is sufficiently dense. In particular, if $M \leq K(\log K)^{2+\varepsilon}$ for some fixed $\varepsilon>0$, then the result of M. Z. Garaev [11 applies to arbitrary sequences $\mathcal{S}$ with $0 \leq s_{k} \leq k^{15 / 14+o(1)}$; however, for shorter sums it loses its power even if the sequence $\mathcal{S}$ is very dense.

Here we consider more general exponential sums and in particular extend the results 11, 12 to a different range of parameters. Roughly speaking, the results of 11, 12 require less averaging but apply to longer sums, while we need more averaging but instead treat shorter (and more general) sums.

More precisely, given two sequences of complex numbers $\Phi=\left(\varphi_{m}\right)_{m=1}^{\infty}$ and $\Psi=\left(\psi_{n}\right)_{n=1}^{\infty}$ we consider the bilinear sums

$$
\sum_{\substack{p \leq M \\ \operatorname{gcd}(a g, p)=1}} \sum_{1 \leq n \leq N} \varphi_{p} \psi_{n} \mathbf{e}_{p}\left(a g^{n}\right), \quad a \in \mathbb{Z},
$$

where the outer summation is taken over all primes $p \leq M$ with $\operatorname{gcd}(a g, p)=1$. Note that we do not request that $a \neq 0$ since for $a=0$ the summation range is empty.

Our method is different from that of M. Z. Garaev 11 and in fact originates from $[3$.

Throughout the paper, the implied constants in the symbols ' $O$ ' and ' $\ll$ ' may depend only on $g$ and two more integer parameters $r$ and $s$ (we recall that $A \ll B$ is equivalent to $A=O(B)$ ). We use the letters $\ell, p$ and $q$ exclusively to denote prime numbers, while $m$ and $n$ always denote positive integers.

\section{MAIN RESUlT}

In the case when some information is available about the growth of the elements of the sequence $\Phi=\left(\varphi_{m}\right)_{m=1}^{\infty}$ (say if $\left|\varphi_{m}\right| \leq 1,1 \leq m \leq M$ ), which is almost always the case, it is easy to see that instead of the sums (1) it is enough to estimate the sums

$$
S_{a}(M, N ; \Psi)=\sum_{\substack{p \leq M \\ \operatorname{gcd}(a g, p)=1}}\left|\sum_{1 \leq n \leq N} \psi_{n} \mathbf{e}_{p}\left(a g^{n}\right)\right|, \quad a \in \mathbb{Z} .
$$

Theorem 1. For any integers $r, s \geq 1$ such that

$$
N^{r+2} \geq M^{2}
$$

the following bound holds uniformly over all $a \in \mathbb{Z}$ :

$$
S_{a}(M, N ; \Psi) \ll F\left(M^{1-1 / 2 r(s+2)} N^{1 / 2+1 / 2 r s}+M^{1+1 /(s+2)}\right),
$$

where

$$
F=\sqrt{\sum_{1 \leq n \leq N}\left|\psi_{n}\right|^{2}}
$$

Proof. Clearly for some complex numbers $\varphi_{m}$ with $\left|\varphi_{m}\right|=1$ for $1 \leq m \leq M$, we have

$$
S_{a}(M, N ; \Psi)=\sum_{\substack{p \leq M \\ \operatorname{gcd}(a g, p)=1}} \varphi_{p} \sum_{1 \leq n \leq N} \psi_{n} \mathbf{e}_{p}\left(a g^{n}\right) .
$$


So, changing the order of summation we obtain

$$
\left|S_{a}(M, N ; \Psi)\right| \leq \sum_{1 \leq n \leq N}\left|\psi_{n}\right|\left|\sum_{\substack{p \leq M \\ \operatorname{gcd}(a g, p)=1}} \varphi_{p} \mathbf{e}_{p}\left(a g^{n}\right)\right| .
$$

Now, using the Cauchy inequality, we obtain

$$
\left|S_{a}(M, N ; \Psi)\right| \leq F U^{1 / 2}
$$

where

$$
\begin{aligned}
U & =\sum_{1 \leq n \leq N}\left|\sum_{\substack{p \leq M \\
\operatorname{gcd}(a g, p)=1}} \varphi_{p} \mathbf{e}_{p}\left(a g^{n}\right)\right|^{2} \\
& =\sum_{\substack{p, q \leq M \\
\operatorname{gcd}(a g, p q)=1}} \varphi_{p} \overline{\varphi_{q}} \sum_{1 \leq n \leq N} \mathbf{e}_{p}\left(a g^{n}\right) \mathbf{e}_{q}\left(-a g^{n}\right) .
\end{aligned}
$$

Let $\mathcal{M}$ be the set of integers $m \leq M^{2}$ which are products of two distinct primes $p<q \leq M$ with $\operatorname{gcd}(a g, p q)=1$. Furthermore, for every $m=p q \in \mathcal{M}$ we define $a_{m}=a(q-p)$; thus

$$
\mathbf{e}_{p}\left(a g^{n}\right) \mathbf{e}_{q}\left(-a g^{n}\right)=\mathbf{e}_{m}\left(a_{m} g^{n}\right)
$$

We also remark that

$$
\operatorname{gcd}\left(a_{m}, m\right)=1
$$

for every $m \in \mathcal{M}$.

Estimating the contribution to $U$ from at most the diagonal terms with $p=q$ trivial as $M N$, we derive

$$
U \leq M N+2 V
$$

where

$$
V=\sum_{m \in \mathcal{M}}\left|\sum_{1 \leq n \leq N} \mathbf{e}_{m}\left(a_{m} g^{n}\right)\right| .
$$

We now remark that for any integer $h \geq 0$ we have

$$
\sum_{1 \leq n \leq N} \mathbf{e}_{m}\left(a_{m} g^{n}\right)=\sum_{1 \leq n \leq N} \mathbf{e}_{m}\left(a_{m} g^{n+h}\right)+O(h) .
$$

Let $H>0$ be an arbitrary integer, to be chosen later. Then, we see from (5) that

$$
V=\frac{W}{H}+O(H \# \mathcal{M})=\frac{W}{H}+O\left(H M^{2}\right)
$$

where

$$
W=\sum_{m \in \mathcal{M}} \sum_{h=1}^{H}\left|\sum_{1 \leq n \leq N} \mathbf{e}_{m}\left(a_{m} g^{n+h}\right)\right| .
$$


By the Hölder inequality, it follows that for any integer $r \geq 1$ we have

$$
\begin{aligned}
W^{r} & \leq H^{r-1}(\# \mathcal{M})^{r-1} \sum_{m \in \mathcal{M}} \sum_{h=1}^{H}\left|\sum_{1 \leq n \leq N} \mathbf{e}_{m}\left(a_{m} g^{n+h}\right)\right|^{r} \\
& =H^{r-1}(\# \mathcal{M})^{r-1} \sum_{m \in \mathcal{M}} \sum_{h=1}^{H} \vartheta_{m, h}\left(\sum_{1 \leq n \leq N} \mathbf{e}_{m}\left(a_{m} g^{n+h}\right)\right)^{r}
\end{aligned}
$$

for some complex numbers $\vartheta_{m, h}$ with $\left|\vartheta_{m, h}\right|=1$.

Now, let $R_{m, k}(K, \lambda)$ denote the number of solutions of the congruence

$$
\sum_{i=1}^{k} g^{w_{i}} \equiv \lambda \quad(\bmod m), \quad 1 \leq w_{1}, \ldots, w_{k} \leq K .
$$

Then

$$
\left(\sum_{1 \leq n \leq N} \mathbf{e}_{m}\left(a_{m} g^{n+h}\right)\right)^{r}=\sum_{\lambda=0}^{p-1} R_{m, r}(N, \lambda) \mathbf{e}_{m}\left(a_{m} \lambda g^{h}\right) .
$$

Therefore, after changing the order of summation (and also using the trivial bound $\# \mathcal{M} \leq M^{2}$, we derive that

$$
W^{r} \leq H^{r-1} M^{2(r-1)} \sum_{m \in \mathcal{M}} \sum_{\lambda=0}^{m-1} R_{m, r}(N, \lambda) \sum_{h=1}^{H} \vartheta_{m, h} \mathbf{e}_{m}\left(a_{m} \lambda g^{h}\right) .
$$

For an integer $s \geq 1$, we write

$$
R_{m, r}(N, \lambda)=\left(R_{m, r}(N, \lambda)^{2}\right)^{1 / 2 s} R_{m, r}(N, \lambda)^{(s-1) / s} .
$$

Using the Hölder inequality for a sum of products of three terms, we have

$$
\begin{aligned}
W^{2 r s} \leq H^{2(r-1) s} & M^{4(r-1) s} \sum_{m \in \mathcal{M}} \sum_{\lambda=0}^{m-1} R_{m, r}(N, \lambda)^{2} \\
& \times\left(\sum_{m \in \mathcal{M}} \sum_{\lambda=0}^{m-1} R_{m, r}(N, \lambda)\right)^{2 s-2} \\
& \times \sum_{m \in \mathcal{M}} \sum_{\lambda=0}^{m-1}\left|\sum_{h=1}^{H} \vartheta_{m, h} \mathbf{e}_{m}\left(a_{m} \lambda g^{h}\right)\right|^{2 s} .
\end{aligned}
$$

Clearly,

$$
\sum_{m \in \mathcal{M}} \sum_{\lambda=0}^{m-1} R_{m, r}(N, \lambda) \leq \# \mathcal{M} N^{r} \leq M^{2} N^{r}
$$

and

$$
\sum_{m \in \mathcal{M}} \sum_{\lambda=0}^{m-1} R_{m, r}(N, \lambda)^{2}=\sum_{m \in \mathcal{M}} T_{m, r}(N),
$$

where $T_{m, k}(K)$ denotes the number of solutions of the congruence

$$
G_{k}\left(w_{1}, \ldots, w_{2 k}\right) \equiv 0 \quad(\bmod m), \quad 1 \leq w_{1}, \ldots, w_{2 k} \leq K,
$$


where

$$
G_{k}\left(w_{1}, \ldots, w_{2 k}\right)=\sum_{i=1}^{2 k}(-1)^{i} g^{w_{i}}
$$

Thus,

$$
\begin{aligned}
W^{2 r s} \leq H^{2(r-1) s} M^{4(r s-1)} N^{2 r(s-1)} \sum_{m \in \mathcal{M}} T_{m, r}(N) & \\
& \times \sum_{m \in \mathcal{M}} \sum_{\lambda=0}^{m-1}\left|\sum_{h=1}^{H} \vartheta_{m, h} \mathbf{e}_{m}\left(a_{m} \lambda g^{h}\right)\right|^{2 s} .
\end{aligned}
$$

Furthermore,

$$
\begin{aligned}
\sum_{\lambda=0}^{m-1}\left|\sum_{h=1}^{H} \vartheta_{m, h} \mathbf{e}_{m}\left(a_{m} \lambda g^{h}\right)\right|^{2 s} & =\sum_{h_{1}, \ldots, h_{2 s}=1}^{H} \prod_{i=1}^{2 s} \vartheta_{m, h_{i}} \sum_{\lambda=0}^{m-1} \mathbf{e}_{m}\left(\lambda G_{s}\left(h_{1}, \ldots, h_{2 s}\right)\right) \\
& \leq \sum_{h_{1}, \ldots, h_{2 s}=1}^{H}\left|\sum_{\lambda=0}^{m-1} \mathbf{e}_{m}\left(\lambda G_{s}\left(h_{1}, \ldots, h_{2 s}\right)\right)\right| \\
& =m T_{m, s}(H) \leq M^{2} T_{m, s}(H) .
\end{aligned}
$$

Hence,

$$
W^{2 r s} \leq H^{2(r-1) s} M^{4 r s-2} N^{2 r(s-1)} \sum_{m \in \mathcal{M}} T_{m, r}(N) \sum_{m \in \mathcal{M}} T_{m, s}(H) .
$$

We note that

$$
\sum_{m \in \mathcal{M}} T_{m, k}(K)=\sum_{w_{1}, \ldots, w_{2 k}=1}^{K} \sum_{\substack{m \in \mathcal{M} \\ m \mid G_{k}\left(w_{1}, \ldots, w_{2 k}\right)}} 1
$$

Clearly, any nonzero value $G_{k}\left(w_{1}, \ldots, w_{2 k}\right) \neq 0$ has at most

$$
\frac{\log \left(2 k g^{K}\right)}{\log 2} \ll K
$$

distinct prime divisors. Thus in this case there are at most $O\left(K^{2}\right)$ values of $m \in$ $\mathcal{M}$ with $m \mid G_{k}\left(w_{1}, \ldots, w_{2 k}\right)$. Thus the total contribution from such terms is $O\left(K^{2 k+2}\right)$.

Furthermore, by the corollary to [15, Lemma 1, Chapter 15], there are at most $2^{k} k ! K^{k}$ integer vectors $\left(w_{1}, \ldots, w_{2 k}\right)$ with $1 \leq w_{1}, \ldots, w_{2 k} \leq K$ and such that $G_{k}\left(w_{1}, \ldots, w_{2 k}\right)=0$. For them we estimate the contribution from the sums over $m \in \mathcal{M}$ trivially as $M^{2}$. Therefore,

$$
\sum_{m \in \mathcal{M}} T_{m, k}(K) \ll K^{2 k+2}+K^{k} M^{2} .
$$

Consequently, inserting (9) into (7), we obtain

$$
W^{2 r s} \ll H^{2(r-1) s} M^{4 r s-2} N^{2 r(s-1)}\left(N^{2 r+2}+N^{r} M^{2}\right)\left(H^{2 s+2}+H^{s} M^{2}\right) .
$$

We now choose

$$
H=\left\lceil M^{2 /(s+2)}\right\rceil
$$


so that $H^{2 s+2}+H^{s} M^{2} \ll H^{s} M^{2}$. Also, recalling that by the condition of the theorem we also have $N^{2 r+2}+N^{r} M^{2} \ll N^{2 r+2}$, we obtain from (10)

$$
W^{2 r s} \ll H^{2 r s-s} M^{4 r s} N^{2 r s+2}
$$

or

$$
W \ll H^{1-1 / 2 r} M^{2} N^{1+1 / r s} .
$$

Substituting this estimate into (6) yields

$$
V \ll H^{-1 / 2 r} M^{2} N^{1+1 / r s}+H M^{2},
$$

which in turn, after substituting into (3), gives

$$
U \ll H^{-1 / 2 r} M^{2} N^{1+1 / r s}+H M^{2}+M N \ll H^{-1 / 2 r} M^{2} N^{1+1 / r s}+H M^{2} .
$$

Inserting this into the inequality (2) and recalling the choice of $H$ produce the desired estimate.

In particular, taking $\Psi$ to be the indicator function of a sequence of integers $\mathcal{S}=\left(s_{k}\right)_{k=1}^{\infty}$, we obtain:

Corollary 2. Let $r, s \geq 1$ be two fixed integers. For any integers $M$ and $N$ with $N \geq M^{2 /(r+2)}$ and any sequence of $K \geq 1$ integers $1 \leq s_{k} \leq N, k=1, \ldots, K$, we have

$$
\sum_{\substack{p \leq M \\ \operatorname{gcd}(a g, p)=1}}\left|\sum_{k=1}^{K} \mathbf{e}_{p}\left(a g^{s_{k}}\right)\right| \ll K^{1 / 2} M^{1-1 / 2 r(s+2)} N^{1 / 2+1 / 2 r s}+M^{1+1 /(s+2)}
$$

uniformly over all $a \in \mathbb{Z}$.

We note that Corollary 2 is nontrivial only if $N^{A} \geq M \geq N^{1+\varepsilon}$ for some fixed $A>1$ and $\varepsilon>0$. In this case, taking a sufficiently large $r$ (to ensure that $N \geq$ $\left.M^{1 / A} \geq M^{2 /(r+2)}\right)$ and then a sufficiently large $s$, we obtain

$$
K^{1 / 2} M^{1-1 / 2 r(s+2)} N^{1 / 2+1 / 2 r s} \leq K^{1 / 2} M N^{1 / 2-\delta}
$$

for some $\delta>0$. Thus if the sequence $s_{1}, \ldots, s_{K}$ is dense enough (for example, $K \geq N^{1-\delta}$ ), then Corollary 2 yields a nontrivial estimate.

On the other hand, the results of M. Z. Garaev 11 require a little less averaging and are nontrivial for smaller values of $M$; however, they become trivial for $M \geq N$.

\section{REMARKS AND OPEN QUESTIONS}

Clearly our estimates can be improved by a power of $\log M$ (as on several occasions when we have used the crude estimate $\# \mathcal{M} \leq M^{2}$ instead of $\# \mathcal{M} \leq$ $\left.M^{2}(\log M)^{-2}\right)$. It is also easy to see that a full analogue of Theorem 1 holds also for the sums

$$
\sum_{\substack{p \leq M \\ \operatorname{gcd}(g, p)=1}} \max _{a=1, \ldots, p-1}\left|\sum_{1 \leq n \leq N} \psi_{n} \mathbf{e}_{p}\left(a g^{n}\right)\right|, \quad a \in \mathbb{Z}
$$

We note that an alternative way to estimate the sums $S_{a}(M, N ; \Psi)$ is via using the estimate due to J. Bourgain and M. Chang [7] directly to estimate the sum over $n$ in (4); see also [5, 6] for further generalisations. However, this approach leads to less explicit estimates and also requires extending the estimates from [5, 6, 7], to incomplete sums (it seems to be very plausible that such an extension is possible, 
though). On the other hand, a clear advantage of this approach is that it can also be used to estimate sums of the type

$$
\sum_{1 \leq m \leq M} \sum_{1 \leq n \leq N} \varphi_{m} \psi_{n} \mathbf{e}_{m}\left(a g^{n}\right), \quad a \in \mathbb{Z}
$$

where the summation is taken over all positive integers $m \leq M$.

Finally, we remark that in [12] one can also find some bounds of multiplicative character sums. It is possible that the methods of [11] apply to multiplicative character sums as well. However the method of this paper does not seem to generalise to such sums. For example, obtaining good estimates on the sums

$$
\sum_{\substack{p \leq M \\ \operatorname{gcd}(a g, p)=1}} \sum_{1 \leq n \leq N} \varphi_{p} \psi_{n}\left(\frac{g^{n}+a}{p}\right), \quad a \in \mathbb{Z},
$$

where $(u / p)$ is the Legendre symbol modulo $p$, remains an open problem.

\section{REFERENCES}

[1] W. Banks, A. Conflitti, J. B. Friedlander and I. E. Shparlinski, 'Exponential sums over Mersenne numbers', Compos. Math., 140 (2004), 15-30. MR2004121 (2004j:11091)

[2] W. Banks, J. B. Friedlander, M. Z. Garaev and I. E. Shparlinski, 'Character sums with exponential functions over smooth numbers', Indag. Math., 17 (2006), 157-168. MR2321378 (2008e:11097)

[3] W. D. Banks, M. Z. Garaev, F. Luca and I. E. Shparlinski, 'Uniform distribution of fractional parts related to pseudoprimes', Canad. J. Math. (to appear).

[4] J. Bourgain, 'Estimates on exponential sums related the Diffie-Hellman distributions', Geom. Funct. Anal., 15 (2005), 1-34. MR2140627 (2006h:11095)

[5] J. Bourgain, 'Exponential sum estimates over subgroups of $\mathbb{Z}_{q}^{*}, q$ arbitrary', J. Anal. Math., 97 (2005), 317-355. MR 2274981 (2007j:11103)

[6] J. Bourgain, 'Exponential sum estimates in finite commutative rings and applications', J. Anal. Math., 101 (2007), 325-355. MR2346549(2008i:11108)

[7] J. Bourgain and M. Chang, 'Exponential sum estimates over subgroups and almost subgroups of $\mathbb{Z}_{Q}^{*}$, where $Q$ is composite with few prime factors', Geom. Funct. Anal., 16 (2006), 327-366. MR2231466 (2007d:11093)

[8] J. Bourgain and M. Z. Garaev, 'On a variant of sum-product estimates and explicit exponential sum bounds in prime fields', Math. Proc. Cambr. Phil. Soc., 146 (2008), 1-21.

[9] J. Bourgain, A. A. Glibichuk and S. V. Konyagin, 'Estimates for the number of sums and products and for exponential sums in fields of prime order', J. Lond. Math. Soc., 73 (2006), 380-398. MR 2225493 (2007e:11092)

[10] M. Dewar, D. Panario and I. E. Shparlinski, 'Distribution of exponential functions with $k$-full exponent modulo a prime', Indag. Math., 15 (2004), 497-503. MR2114933 (2005k:11166)

[11] M. Z. Garaev, 'The large sieve inequality for the exponential sequence $\lambda^{\left[O\left(n^{15 / 14+o(1)}\right)\right]}$ modulo primes', Canad. J. Math. (to appear).

[12] M. Z. Garaev and I. E. Shparlinski, 'The large sieve inequality with exponential functions and the distribution of Mersenne numbers modulo primes', Intern. Math. Res. Notices, 2005:39 (2005), 2391-2408. MR2181356 (2006i:11108)

[13] S. V. Konyagin and I. E. Shparlinski, Character sums with exponential functions and their applications, Cambridge Univ. Press, Cambridge, 1999. MR.1725241 (2000h:11089)

[14] N. M. Korobov, 'On the distribution of digits in periodic fractions', Matem. Sbornik, 89(131) (1972), 654-670 (in Russian). MR0424660 (54:12619)

[15] A. G. Postnikov, Ergodic aspects of the theory of congruences and of the theory of Diophantine approximations, Trudy Mat. Inst. Steklov, vol. 82, 1966 (Russian); translated by the Amer. Math. Soc., Providence, R.I., 1967. MR 0214561 (35:5410)

[16] I. E. Shparlinski, Cryptographic applications of analytic number theory, Birkhäuser Verlag, Basel, 2003. MR:1954519 (2004h:94049) 
[17] I. E. Shparlinski, 'Distribution of exponential functions with squarefull exponent in residue rings', Indag. Math., 15 (2004), 283-289. MR2071861 (2005h:11183)

[18] A. Topuzoğlu and A. Winterhof, 'Pseudorandom sequences', Topics in Geometry, Coding Theory and Cryptography, Springer, Dordrecht, 2007, 135-166. MR:2278037(2007m:11106)

Department of Computing, Macquarie University, Sydney, NSW 2109, Australia

E-mail address: igor@ics.mq.edu.au 